\title{
A Multi-Level Multi-Objective Quadratic Programming Problem with Fuzzy Parameters on Objective Functions \\ O.E.Emam. A.M.Abdo. N.M. Bekhit.
}

Information Systems Department, Faculty of Computers and Information,Helwan University, Cairo,Egypt.

$\frac{\text { Emam_o_e@yahoo.com }}{\text { Amanyabdo_80@yahoo.com }}$

\section{ABSTRACT}

This paper proposes analgorithm to solve multi-level multi-objective quadratic programming problemwith fuzzy parameters in the objective functions, This algorithm uses the tolerance membership function conceptsand multi-objective optimization at each level to develop a fuzzy Max-Min decision model for generating satisfactory solution after applying linear rankingmethod ontrapezoidal fuzzy numbers in the objective functions, An illustrative example is included to explain the results.

\section{Keywords}

Multi-Level Programming,Trapezoidal Fuzzy Numbers, linear Ranking Methods, Fuzzy approach.

\section{Academic Discipline And Sub-Disciplines}

Computers \& Technology

Management \& Information Technology

\section{TYPE (METHOD/APPROACH)}

Multi- Objective Non-linear Programming, Fuzzy programming techniques, Quadratic programming.

\section{INTRODUCTION}

Multi-level programming (MLP) techniques are developed to solve decentralized problems that contain multiple decision-makers in hierarchical organization, where each unit or department independently seeks its own interest, but is affected by the actions of other unit through externalities.Three level programming is a class of Multi-level programming problem in which there are three independent decision-makers. The field of multi-level programming which defines the art and science of making such decisions studied in $[2,3$, and 4$]$.

Fuzzy approach uses the concept of tolerance membership to develop a fuzzy max-min decisionModel for generating Pareto optimal (satisfactory) solution [1 and 3]

In [3] Osman et al. presented the concepts of tolerance membership function and multi-objective optimization at each level to develop a fuzzy max-min decision model for generating (satisfactory) solution for three level multi-objective programming problem.

Recently several linear or nonlinear programming problems with fuzzy parameters on objective functions and their solution method have been presented, such as, in [4, 5, 6,7, 8, 9, 10and11]. In [4] Under the rules of simplex technique and the operations on trapezoidal fuzzy numbersEmamet al. ,suggested a new solution method to solve bi-level linear fractional integer programming problem with trapezoidal fuzzy numbers in the objective functions of the two levels .In [5]Dashetal. aimed to present a method in which a fuzzy multi objective nonlinear programming problem is reduced to crisp using ranking function and then the crisp problem is solved by fuzzy programming technique. In [6]Nasseri defined a quadratic programming problem with trapezoidal and/or triangularfuzzy numbers in the cost coefficients,constraint coefficients, and right-hand sides then used linear ranking method to solve the problem.

\section{PROBLEM FORMULATION AND SOLUTION CONCEPT}

Let $x_{i} \in R^{n},(i=1,2,3)$, be a vector of variables which indicates the first decision level's choice, thesecond decision level's choice and the third decision level's choice andF $: R^{n} \rightarrow R^{N_{t}}(i=1,2,3)$, be the first level objective function, the second level objective function and the third level objective function, respectively. Assume thatthe first level decision maker is (FLDM),the secondlevel decision maker is(SLDM) and the third level decision maker is(TLDM). $N_{1}, N_{2}$ and $N_{3} \geq 2$, the FLDM, SLDM, and TLDM have $N_{1}, N_{2}$ and $N_{3}$ objective functions, respectively.Let $G$ be the set of feasible choices $\left\{\left(x_{1}, x_{2}, x_{3}\right)\right\}$. Therefore a(MLMOQPP) with fuzzy parameters in the objective functionsmay be formulated as follows: 
$\left[1^{\text {st }}\right.$ level $]$

$\max _{1}(\bar{x}, \tilde{q}, \tilde{C})=\max x_{x_{1}}\left[\left(\frac{1}{2} \sum_{i=1}^{n} \sum_{j=1}^{n} \tilde{q}_{i j} x_{i} x_{j}+\sum_{j=1}^{n} \tilde{C}_{j} x_{j}\right), \ldots, f_{1 N_{1}}\left(\frac{1}{2} \sum_{i=1}^{n} \sum_{j=1}^{n} \tilde{q}_{i j} x_{i} x_{j}+\sum_{j=1}^{n} \tilde{C}_{j} x_{j}\right)\right]$

$\left[2^{\text {nd }}\right.$ level $]$

Where $\mathbf{x}_{2}, \mathbf{x}_{3}$ solve

$$
\max _{2}(\overline{\mathrm{x}}, \tilde{\mathrm{q}}, \tilde{\mathrm{C}})=\max _{\mathrm{x}_{2}}\left[\left(\frac{1}{2} \sum_{\mathrm{i}=1}^{\mathrm{n}} \sum_{\mathrm{j}=1}^{\mathrm{n}} \tilde{\mathrm{q}}_{\mathrm{ij}} \mathrm{x}_{\mathrm{i}} \mathrm{x}_{\mathrm{j}}+\sum_{\mathrm{j}=1}^{\mathrm{n}} \tilde{\mathrm{C}}_{\mathrm{j}} \mathrm{x}_{\mathrm{j}}\right), \ldots, \mathrm{f}_{2 \mathrm{~N}_{2}}\left(\frac{1}{2} \sum_{\mathrm{i}=1}^{\mathrm{n}} \sum_{\mathrm{j}=1}^{\mathrm{n}} \tilde{\mathrm{q}}_{\mathrm{ij}} \mathrm{x}_{\mathrm{i}} \mathrm{x}_{\mathrm{j}}+\sum_{\mathrm{j}=1}^{\mathrm{n}} \tilde{\mathrm{C}}_{\mathrm{j}} \mathrm{x}_{\mathrm{j}}\right)\right]
$$

Where $\mathbf{x}_{\mathbf{3}}$ solves

$\left[3^{\text {rd level }]}\right.$

$$
\max _{3}(\bar{x}, \tilde{\mathrm{q}}, \tilde{\mathrm{C}})=\max _{\mathrm{x}_{3}}\left[\left(\frac{1}{2} \sum_{\mathrm{i}=1}^{\mathrm{n}} \sum_{\mathrm{j}=1}^{\mathrm{n}} \tilde{\mathrm{q}}_{\mathrm{ij}} \mathrm{x}_{\mathrm{i}} \mathrm{x}_{\mathrm{j}}+\sum_{\mathrm{j}=1}^{\mathrm{n}} \tilde{\mathrm{C}}_{\mathrm{j}} \mathrm{x}_{\mathrm{j}}\right), \ldots, \mathrm{f}_{3 \mathrm{~N}_{3}}\left(\frac{1}{2} \sum_{\mathrm{i}=1}^{\mathrm{n}} \sum_{\mathrm{j}=1}^{\mathrm{n}} \tilde{\mathrm{q}}_{\mathrm{ij}} \mathrm{x}_{\mathrm{i}} \mathrm{x}_{\mathrm{j}}+\sum_{\mathrm{j}=1}^{\mathrm{n}} \tilde{\mathrm{C}}_{\mathrm{j}} \mathrm{x}_{\mathrm{j}}\right)\right]
$$

Subject to:

$G:\left\{(\bar{x}) \mid g_{i}(\bar{x}) \leq 0, i=1,2, \ldots, m\right\}, \bar{x}=\left(x_{1}, x_{2}, x_{3}\right) \in R^{n_{1}+n_{2}+n_{3}}$

Where $F_{i}(\bar{x}, \tilde{\mathrm{q}}, \tilde{\mathrm{C}})$, is a multi-level multi-objective quadratic programming problemwith fuzzy parameters in the objective functions.

\section{Definition 1 [3]:}

Let $G_{1}, G_{2}, G_{3}$ be the feasible regions of FLDM, SLDM and TLDM, respectively. For any $\left(x_{1} \in G_{1}=\left\{x_{1} \mid\left(x_{1}, x_{2}, x_{3}\right)\right\}\right.$. $\left.\left.\in G_{1}\right\}\right)$ given by FLDM, and $\left(x_{2} \in G_{2}=\left\{x_{2} \mid\left(x_{1}, x_{2}, x_{3} \in G_{2}\right\}\right)\right.$ given by SLDM, if the decision-makingvariable $\left(x_{3} \in G_{3}=\left\{x_{3} \mid\left(x_{1}, x_{2}, x_{3}\right) \in G_{3}\right\}\right)$ is the optimal solution of the TLDM, then $\left(x_{1}, x_{2}, x_{3}\right)$ is a feasible solution of the(MLMOQPP) with fuzzy parameters in the objective functions.

\section{Definition 2[3]:}

If $\left(x_{1}^{*}, x_{2}^{*}, x_{3}^{*}\right)$ is a feasible solution of the (MLMOQPP) with fuzzy parameters in the objective functions(1)-(4); no other feasible solution $\left(x_{1}, x_{2}, x_{3}\right) \in G$ exists, suchthat $f_{1 i}\left(x_{1}^{*}, x_{2}^{*}, x_{3}^{*}\right) \leq f_{1 i}\left(x_{1}, x_{2}, x_{3}\right)$, with at least one $\left(i=1,2, . ., K_{i}\right)$; $\operatorname{so}\left(x_{1}^{*}, x_{2}^{*}, x_{3}^{*}\right)$ is the optimal solutions of the (MLMOQPP) with fuzzy parameters in the objective functions.

\section{RANKING METHOD}

To solve (MLMOQPP) with fuzzy parameters in the objective functions a linear ranking method technique is used to convert fuzzy number form into equivalent crisp form.

\section{Definition 3.1[10]:}

$\operatorname{If}(\widetilde{\mathrm{A}})=(\mathrm{a}, \mathrm{b}, \mathrm{c}, \mathrm{d}) \in \mathrm{F}(\mathrm{R})$, then a linear ranking function is defined as $\Re(\widetilde{\mathrm{A}})=\mathrm{a}+\mathrm{b}+\frac{1}{2}(\mathrm{~d}-\mathrm{c})$

\section{Definition 3.2[10]:}

$\widetilde{A}\left(a_{1}, b_{1}, c_{1}, d_{1}\right), \widetilde{B}\left(a_{2}, b_{2}, c_{2}, d_{2}\right)$ are two trapezoidal fuzzy numbers and $x \in R$. Ranking function is a convenient method for comparing the fuzzy numbers which is a map from $F(R)$ into the real line. So, the orders on $F(R)$ as follow:

1. $\widetilde{\mathrm{A}} \geq \widetilde{\mathrm{B}}$ if and only if $\mathfrak{R}(\widetilde{\mathrm{A}}) \geq \mathfrak{R}(\widetilde{\mathrm{B}})$.

2. $\widetilde{\mathrm{A}}>\widetilde{\mathrm{B}}$ if and only if $\Re(\widetilde{\mathrm{A}})>R(\widetilde{\mathrm{B}})$.

3. $\widetilde{\mathrm{A}}=\widetilde{\mathrm{B}}$ if and only if $\mathfrak{R}(\widetilde{\mathrm{A}})=\mathfrak{R}(\widetilde{\mathrm{B}})$.

Where $\tilde{A}$ and $\tilde{B}$ are in $\mathrm{F}(\mathrm{R})$.

Nowafter applying linear ranking methodthe problem will be formulated as follow: 
$\left[1^{\text {st }}\right.$ level $]$

$$
\max _{1}\left(x_{1}, x_{2}, x_{3}\right)=\max _{x_{1}}\left[\left(\frac{1}{2} \sum_{i=1}^{n} \sum_{j=1}^{n} q_{i j} x_{i} x_{j}+\sum_{j=1}^{n} C_{j} x_{j}\right), \ldots, f_{1 N_{1}}\left(\frac{1}{2} \sum_{i=1}^{n} \sum_{j=1}^{n} q_{i j} x_{i} x_{j}+\sum_{j=1}^{n} C_{j} x_{j}\right)\right]
$$

Where $x_{2}, x_{3}$ solve

$\left[2^{\text {nd }}\right.$ level $]$

$$
\max _{2}\left(x_{1}, x_{2}, x_{3}\right)=\max _{x_{2}}\left[\left(\frac{1}{2} \sum_{i=1}^{n} \sum_{j=1}^{n} q_{i j} x_{i} x_{j}+\sum_{j=1}^{n} C_{j} x_{j}\right), \ldots, f_{2 N_{2}}\left(\frac{1}{2} \sum_{i=1}^{n} \sum_{j=1}^{n} q_{i j} x_{i} x_{j}+\sum_{j=1}^{n} C_{j} x_{j}\right)\right]
$$

Where $x_{3}$ solves

$\left[3^{\text {rd }}\right.$ level $]$

$$
\max _{2}\left(x_{1}, x_{2}, x_{3}\right)=\max _{x_{3}}\left[\left(\frac{1}{2} \sum_{i=1}^{n} \sum_{j=1}^{n} q_{i j} x_{i} x_{j}+\sum_{j=1}^{n} C_{j} x_{j}\right), \ldots, f_{3 N_{3}}\left(\frac{1}{2} \sum_{i=1}^{n} \sum_{j=1}^{n} q_{i j} x_{i} x_{j}+\sum_{j=1}^{n} C_{j} x_{j}\right)\right]
$$

Subject to:

$G:\left\{(\bar{x}) \mid g_{i}(\bar{x}) \leq 0, i=1,2, \ldots, m\right\}, \bar{x}=\left(x_{1}, x_{2}, x_{3}\right) \in R^{n_{1}+n_{2}+n_{3}}$

\section{FUZZY APPROACH FOR SOLVING (MLMOQPP)}

To solve the (MLMOQPP) by using fuzzy approach, first the satisfactory solution that is acceptable for the FLDM is obtained, then the FLDM's decision variables and goals with some leeway are given to the SLDM to seek the satisfactory solution according to him/her, then the SLDM's decision variables and goals with some leeway are given to the TLDM to seek the satisfactory solution according to him/her and finally to arrive at the solution which is closest to the optimal solution of the FLDM.[3]

\subsection{The problem ofthe FLDM.}

The problem of theFLDM is solved individually;FLDM'soptimal solution is achieved by applying the following steps, firstby obtaining the best and the worst solutions oftheProblem

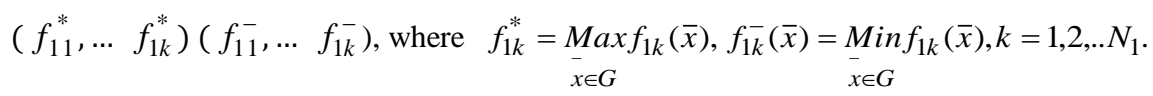

Second Usingthe value of $\left(f_{1 k}^{*}, f_{1 k}^{-}\right)$to build membership functions as follows:

$$
\mu_{f_{1 k}}\left[f_{1 k}(\bar{x})\right]=\left\{\begin{array}{lll}
1 & \text { if } & f_{1 k}(\bar{x})>f_{1 k}^{*} \\
\frac{f_{1 k}(\bar{x})-f_{1 k}^{-}}{f_{1 k}^{*}-f_{1 k}^{-}} & \text {if } & f_{1 k}^{-} \leq f_{1 k}(\bar{x}) \leq f_{1 K}^{*} \\
0 & \text { if } & f_{1 k}^{-} \geq f_{1 k}(\bar{x}) \\
& & k=1,2, \ldots, N_{1}
\end{array}\right.
$$

Third by solving the Tchebycheff problem as follow: 
$\operatorname{Max} \lambda$

Subject to:

$$
\begin{aligned}
& \{\bar{x} \in G, \\
& \mu_{f_{1 k}}\left[f_{1 k}(\bar{x})\right] \geq \lambda, k=1,2, \ldots, N_{1}, \\
& \lambda \in[0,1]\}
\end{aligned}
$$

Whose solution is assumed to be: $\left[x_{1}^{F}, x_{2}^{F}, x_{3}^{F^{*}}, f_{1 k}^{F}, k=1,2, \ldots, N, \lambda^{F}\right] \quad$ (satisfactory level).

Now the SLDM and TLDM do the same action like the FLDM till they obtains their optimal solutions as $\left[x_{1}^{S}, x_{2}^{S}, x_{3}^{S}, f_{2 q}^{S}, q=1,2, \ldots, N, \beta^{S}\right]$ and $\left[x_{1}^{t}, x_{2}^{t}, x_{3}^{t}, f_{2 r}^{t}, r=1,2, \ldots, N, \gamma^{t}\right]$

Now the solution of the three level decision makers is disclosed. However, three solutions are usually different because of nature between three levels objective functions.

The FLDM knows that using the optimal decisions $x_{1}^{F}$ as a control factors for the SLDM are not practical. It is more reasonable to have some tolerance that gives the SLDM an extent feasible region to search for his/her optimal solution, and reduce searching time or interactions, also the SLDM do the same action with the TLDM. In this way, the range of decision variables $x_{1}, x_{2}$ should be around $x_{1}^{F}, x_{2}^{S}$ with maximum tolerance $t_{1}, t_{2}$ and the following membership function specify $x_{1}^{F}, x_{2}^{S}$ as:

$\mu_{x_{1}}\left(x_{1}\right)= \begin{cases}\frac{x_{1}-\left(x_{1}^{F}-t_{1}\right)}{t_{1}} & x_{1}^{F}-t_{1} \leq x_{1} \leq x_{1}^{F} \\ \frac{\left(x_{1}^{F}+t_{1}\right)-x_{1}}{t_{1}} & x_{1}^{F} \leq x_{1} \leq x_{1}^{F}+t_{1}\end{cases}$

$\mu_{x_{2}}\left(x_{2}\right)= \begin{cases}\frac{x_{2}-\left(x_{2}^{S}-t_{2}\right)}{t_{1}} & x_{2}^{S}-t_{2} \leq x_{2} \leq x_{2}^{S} \\ \frac{\left(x_{2}^{S}+t_{2}\right)-x_{2}}{t_{2}} & x_{2}^{S} \leq x_{2} \leq x_{2}^{S}+t_{2}\end{cases}$

The FLDM goal may be reasonably consider all $f_{1 k} \geq f_{1 k}^{F} k=1,2, \ldots N_{1}$ are absolutely acceptable and $f_{1 k}$

$<f_{1 k}^{\prime}=f_{1 k}\left(x_{1}^{S}, x_{2}^{S}, x_{3}^{S}\right), k=1,2, \ldots N_{1}$ are absolutely unacceptable, and that the preference with $\left[f_{1 k}^{F}, f_{1 k}^{\prime}, \mathrm{k}=1,2, \ldots N_{1}\right]$ is linearly increasing. This is due to the fact that the SLDM obtained the optimum at $\left(x_{1}^{S}, x_{2}^{S}, x_{3}^{S}\right)$ which in turn provides the FLDM the objective function values $f_{1 k}^{\prime}$, makes any $f_{1 k}^{F}, f_{1 k}^{\prime}, \mathrm{k}=1,2, \ldots N_{1}$, unattractive in practice, The following membership functions of the FLDM can be stated as

$\mu_{f_{1 k}}^{\prime}\left[f_{1 k}(\bar{x})\right]= \begin{cases}1 & \text { if } f_{1 k}>f_{1 k}^{F}(x) \\ \frac{f_{1 k}(\bar{x})-f_{1 k}^{\prime}}{f_{1 k}^{F}-f_{1 k}^{\prime}} & \text { if } f_{1 k}^{\prime} \leq f_{1 k}(x) \leq f_{1 K}^{F} \\ 0 & \text { if } f_{1 k}(x) \geq f_{1 k}^{\prime}\end{cases}$

Second, the SLDM goal may reasonably consider all $f_{2 q} \geq f_{2 q}^{S} \geq q=1,2, \ldots N_{2}$ are absolutely acceptable and $f_{2 q}<f_{2 q}^{\prime}=$ $f_{2 q}\left(x_{1}^{T}, x_{2}^{T}, x_{3}^{T}\right), q=1,2, \ldots N_{1}$ are absolutely unacceptable, and that the preference with $\left[f_{2 q}^{S}, f_{2 q}^{\prime}, \mathrm{q}=1,2, \ldots N_{2}\right]$ is linearly 
increasing. This is due to the fact that the TLDM obtained the optimum at $\left(x_{1}^{T}, x_{2}^{T}, x_{3}^{T}\right)$ which in turn provides the SLDM the objective function values $f_{2 q}^{\prime}$, makes any $\left[f_{2 q}^{S}, f_{2 q}^{\prime}, \mathrm{q}=1,2, \ldots N_{2}\right]$, unattractive in practice, The following membership functions of the SLDM can be stated as

$\mu_{f_{2 q}}^{\prime}\left[f_{2 q}(\bar{x})\right]= \begin{cases}1 & \text { if } f_{2 q}>f_{2 q}^{S}(x) \\ \frac{f_{2 q}(\bar{x})-f_{2 q}^{\prime}}{f_{2 q}^{S}-f_{2 q}^{\prime}} & \text { if } f_{2 q}^{\prime} \leq f_{2 q}(x) \leq f_{2 q}^{S} \\ 0 & \text { if } f_{2 q}(x) \geq f_{2 q}^{\prime}\end{cases}$

Third, the TLDM may be willing to build a membership function for his/her objective functions, so that he/she can rate the satisfaction of each potential solution. In this way, the TLDM has the following membership functions for his/her goals:

$\mu_{f_{3 r}}^{\prime}\left[f_{3 r}(\bar{x})\right]= \begin{cases}1 & \text { if } f_{3 r}>f_{3 r}^{T}(x) \\ \frac{f_{3 r}(\bar{x})-f_{3 r}^{\prime}}{f_{3 r}^{T}-f_{3 r}^{\prime}} & \text { if } f_{3 r}^{\prime} \leq f_{3 r}(x) \leq f_{3 r}^{T} \\ 0 & \text { if } f_{3 r}(x) \geq f_{3 r}^{\prime}\end{cases}$

Finally, in order to generate the satisfactory solution, which is also a Pareto optimal solution with overall satisfaction for all DMs, theTchebycheff problem can be solved as follow:

$\operatorname{Max} \delta$

Subject to:

$\{\bar{x} \in G$,

$\mu\left(x_{i}\right) \geq \delta$,

$\mu_{f_{1 k}}\left[f_{1 k}(\bar{x})\right] \geq \delta,\left(k=1,2, \ldots, N_{1}\right)$,

$\mu_{f_{2 q}}\left[f_{2 q}(\bar{x})\right] \geq \delta,\left(q=1,2, \ldots, N_{2}\right)$,

$\mu_{f_{3 r}}\left[f_{3 r}(\bar{x})\right] \geq \delta,\left(r=1,2, \ldots, N_{3}\right)$,

$i=1,2$.

$\delta \in[0,1]\}$.

\section{AN ALGORITHM}

In this section an algorithm is presented to solve (MLMOQPP) with fuzzy parameters in the objective functions, the algorithm is illustrated in the following series steps:

Step 1: Compute $\Re(\tilde{A})$ for all the coefficients ofthe (MLMOQPP) with fuzzy number in the objective functions (1) - (3), where $\tilde{A}$ is a trapezoidal fuzzy number.

Step 2: Convert the (MLMOQPP) with fuzzy number in the objective functions (1) - (3)from the fuzzy form to the crisp form.

Step 3: Formulate the (MLMOQPP) (6) - (8).

Step 4: Use fuzzy approachto solve the (MLMOQPP).

Step 5: The FLDM finds the individual best solutions $f_{1 k}^{*}$ and individual worst $f_{1 k}^{-}$for each objective of the FLDM.

Step 6: State membership functions of the FLDM, $\mu_{f_{1 k}}\left\lfloor f_{1 k}(\bar{x})\right\rfloor$

Step 7: Solve the Tchebycheff problem of the FLDM.

Step 8: Obtain satisfactory level of FLDM $x_{1}^{F}, x_{2}^{F}, x_{3}^{F}, f_{1 k}^{F}, k=1,2, \ldots, N, \lambda^{F}$.

Step 9: The SLDM finds the individual best solutions $f_{2 q}^{*}$ and individual worst $f_{2 q}^{-}$for each objective of the SLDM. 
Step 10: State membership functions of the SLDM, $\mu_{f_{2 q}}\left[f_{2 q}(\bar{x})\right]$.

Step 11: Solve the Tchebycheff problem of the SLDM.

Step 12: Obtain satisfactory level of $\operatorname{SLDM}\left[x_{1}^{S}, x_{2}^{S}, x_{3}^{S}, f_{2 q}^{S}, q=1,2, \ldots, N, \beta^{S}\right]$.

Step 13: The TLDM finds the individual best solutions $f_{3 r}^{*}$ and individual worst $f_{3 r}^{-}$for each objective of the TLDM.

Step 14: State membership functions of the TLDM $\mu_{f_{3 r}}\left\lfloor f_{3 r}(\bar{x})\right\rfloor$.

Step 15: Solve the Tchebycheff problem of the TLDM.

Step 16: Obtain satisfactory level of TLDM $\left[x_{1}^{t}, x_{2}^{t}, x_{3}^{t}, f_{2 r}^{t}, r=1,2, \ldots, N, \gamma^{t}\right]$

.Step 17: Set $t_{1}, t_{2}$ then calculate $\mu_{x_{1}}\left(x_{1}\right), \mu_{x_{2}}\left(x_{2}\right)$

Step 18: state membership function $\mu_{f_{1 k}^{\prime}}\left\lfloor f_{1 k}(\bar{x})\right\rfloor, \mu_{f_{2 q}}^{\prime}\left\lfloor f_{2 q}(\bar{x})\right\rfloor, \mu_{f_{3 r}}^{\prime}\left\lfloor f_{3 r}(\bar{x})\right\rfloor$

Step 19: Solve the Tchebycheff problem for all decision makers problem.

Step 20: If $\delta<0.5$, decrease tolerance value $t_{1}$, $t_{2}$, then go to step 17 , otherwise go to step 21 .

Step 21: A compromise solution $X^{0}$ of the (MLMOQP) problem is obtained and $\delta$ is overall satisfaction for all decision - makers.

\subsection{A flowchart:}

A flowchart to explain the suggested algorithm is described as follow: 


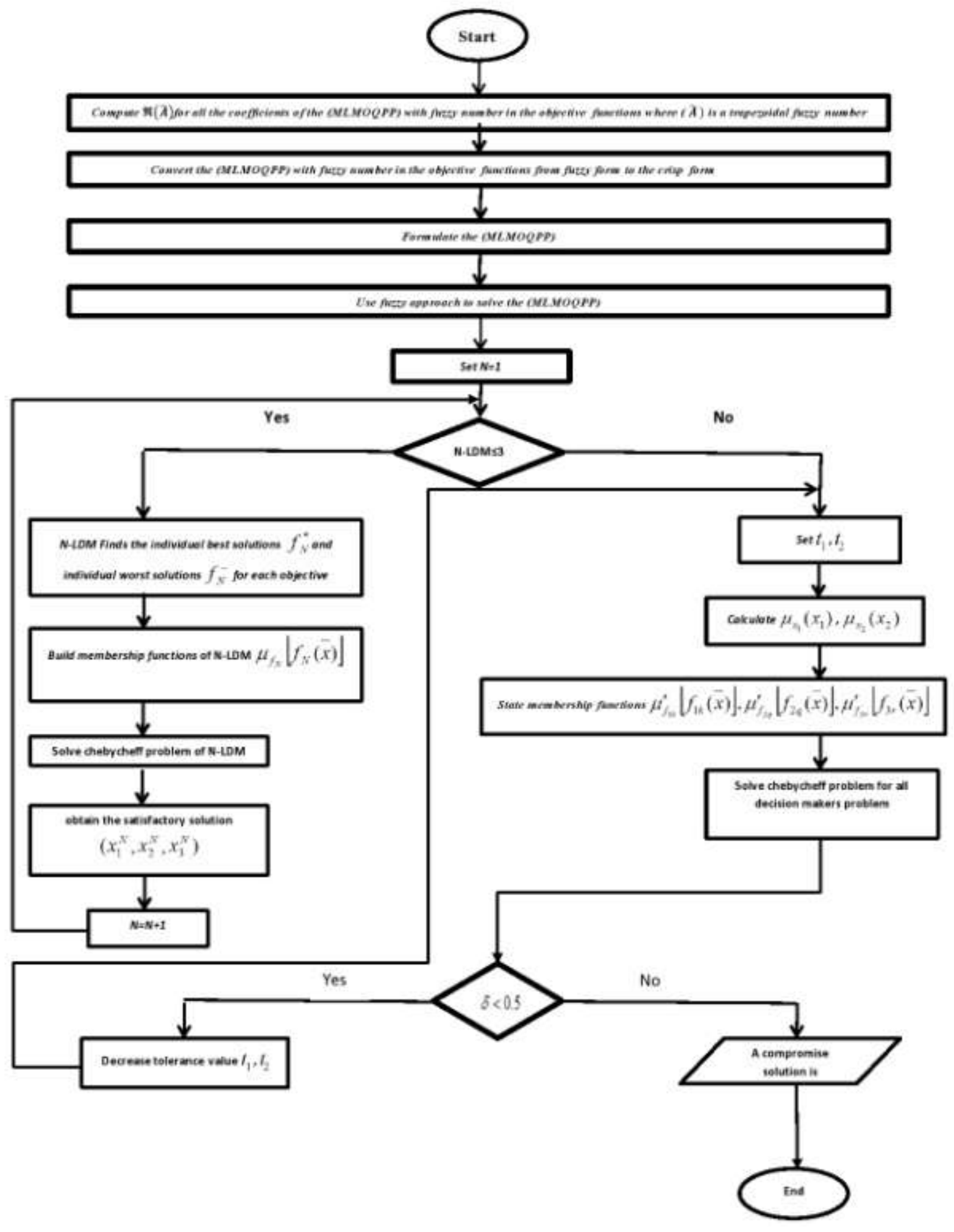

Fig1: An algorithm for solving the (MLMOQPP) with fuzzy number in the objective functions. 


\section{NUMERICAL EXAMPLE}

$\left[1^{\text {st }}\right.$ level $]$

$$
\begin{gathered}
\max F_{1}\left(x_{1}, x_{2}, x_{3}\right)=\max \quad\left[3(1,3,2,4) x_{1}^{2}+x_{2}^{2}+x_{3} \quad, \quad 4(1,2,4,1) x_{1}^{2}+3 x_{2}^{2}\right] \\
x_{1} \quad x_{1} \quad \text { Where } x_{2}, x_{3} \text { solves }
\end{gathered}
$$

$\left[2^{\text {nd }}\right.$ level $]$

$$
\begin{gathered}
\max _{2}\left(x_{1}, x_{2}, x_{3}\right)=\max _{x_{2}}\left[x_{1}^{2}+3 x_{2}^{2} \quad, \quad 2 x_{1}^{2}+(2,4,6,8) x_{2}^{2}+3 x_{3}\right] \\
\quad \text { Where } x_{3} \text { solves }
\end{gathered}
$$

$\left[3^{\text {rd }}\right.$ level $]$

$$
\max _{3}\left(x_{1}, x_{2}, x_{3}\right)_{x_{3}}=\max _{x_{3}}\left[\Phi_{1}+x_{2}+3(1,3,2,4) x_{3}^{2}, \quad 2 x_{1}^{2}+x_{2}^{2}+2(3,4,5,6) x_{3}^{2}\right]
$$

Subject to

$\left(x_{1}, x_{2}, x_{3}\right) \in \mathrm{G}=$

$$
\begin{aligned}
\left\{\left(x_{1}, x_{2}, \quad x_{3}\right)\right. & \\
x_{1}+x_{2} & +x_{3} \leq 5 \\
x_{1}+2 x_{2} & +x_{3} \leq 10 \\
2 x_{1}+x_{2} & +x_{3} \leq 14
\end{aligned}
$$

$\left.x_{1}, x_{2}, x_{3} \geq 0\right\}$

Firstly, by using equation (5)all the coefficients ofthe problem are computed so the problem is convertedfrom the fuzzy form to the crisp formthen the problem is formulated as follow:

$\left[1^{\text {st }}\right.$ level $]$

$$
\begin{aligned}
& \max F_{1}\left(x_{1}, x_{2}, x_{3}\right)=\max \quad\left[15 x_{1}^{2}+x_{2}^{2}+x_{3}, 6 x_{1}^{2}+3 x_{2}^{2}\right] \\
& \text { Where } x_{2} \text { solves }
\end{aligned}
$$

$\left[2^{\text {nd }}\right.$ level $]$

$$
\begin{aligned}
\max _{2}\left(x_{1}, x_{2}, x_{3}\right) & =\max _{x_{2}}\left[x_{1}^{2}+3 x_{2}^{2}, \quad 2 x_{1}^{2}+7 x_{2}^{2}+3 x_{3}\right] \\
x_{2} & \quad \text { Where } x_{3} \text { solves }
\end{aligned}
$$

$\left[3^{\text {rd }}\right.$ level $]$

$$
\max _{3}\left(x_{1}, x_{2}, x_{3}\right)_{x_{3}}=\max _{x_{3}}\left[x_{1}+x_{2}+15 x_{3}^{2} \quad 2 x_{1}^{2}+x_{2}^{2}+15 x_{3}^{2}\right]
$$

Subject to

$$
\begin{aligned}
& \left(x_{1}, \quad x_{2}, \quad x_{3}\right) \in \mathrm{G}= \\
& \left\{\left(x_{1}, x_{2}, x_{3}\right)\right. \\
& x_{1}+x_{2}+x_{3} \leq 5 \\
& x_{1}+2 x_{2}+x_{3} \leq 10 \\
& 2 x_{1}+x_{2}+x_{3} \leq 14
\end{aligned}
$$

$\left.x_{1}, x_{2}, x_{3} \geq 0\right\}$

Secondly, by using fuzzy approach, TheFLDM solves his/her problem as follows:

$\left[1^{\text {st }}\right.$ level $]$

$$
\max F_{1}\left(x_{1}, x_{2}, x_{3}\right)=\max \left[15 x_{1}^{2}+x_{2}^{2}+x_{3}, \quad 6 x_{1}^{2}+3 x_{2}^{2}\right]
$$

Subject to

$$
\left(x_{1}, x_{2}, x_{3}\right) \in \mathrm{G}=\quad\left\{\begin{array}{r}
\left(x_{1}, x_{2}, x_{3}\right) \\
x_{1}+x_{2}+x_{3} \leq 5
\end{array}\right.
$$


$\left.x_{1}, x_{2}, x_{3} \geq 0\right\}$

$$
\begin{gathered}
x_{1}+2 x_{2}+x_{3} \leq 10 \\
2 x_{1}+x_{2}+x_{3} \leq 14
\end{gathered}
$$

By solving equation $(9),\left(f_{11}^{*}, f_{12}^{*}\right)=(375,150),\left(f_{11}^{-}, f_{12}^{-}\right)=(-.18,-.19)$, thenequation $(10)$ and then equation $(11)$

$\operatorname{Max} \lambda$,

Subject to: \{

$\left(x_{1}, x_{2}, x_{3}\right) \in G$

$\lambda \in[0,2]$,

$15 x_{1}^{2}+x_{2}^{2}+x_{3}-375.18 \lambda>=-.18$,

$\left.6 x_{1}^{2}+3 x_{2}^{2}-150.19 \lambda>=-0.19\right\}$

Whose solution is assumed to be:

$\left(x_{1}^{F}, x_{2}^{F}, x_{3}^{F}\right)=(2.60,0.67,1.23),\left(f_{11}^{F}, f_{12}^{F}\right)=(103.07,91.90), \lambda=0.2$

The SLDM solves his/her problem exactly like FLDM as follow:

$\left[2^{\text {nd }}\right.$ level $]$

$$
\max _{2}\left(x_{1}, x_{2}, x_{3}\right)=\max _{x_{2}}\left[x_{1}^{2}+3 x_{2}^{2}, \quad 2 x_{1}^{2}+7 x_{2}^{2}+3 x_{3}\right]
$$

Subject to:

$\left(x_{1}, x_{2}, x_{3}\right) \in \mathrm{G}=\left\{\left(x_{1}, \quad x_{2}, x_{3}\right)\right.$

$\left.x_{1}, x_{2}, x_{3} \geq 0\right\}$

$$
\begin{array}{cl}
x_{1}+x_{2} & +x_{3} \leq 5 \\
x_{1}+2 x_{2} & +x_{3} \leq 10 \\
2 x_{1}+x_{2} & +x_{3} \leq 14
\end{array}
$$

$\left(f_{21}^{*}, f_{22}^{*}\right)=(75,175) \cdot\left(f_{21}^{-}, f_{22}^{-}\right)=(-0.16,-0.19)$

$\operatorname{Max} \beta$,

Subject to :\{

$\left(x_{1}, x_{2}, x_{3}\right) \in \mathrm{G}$,

$\beta \in[0,0.1]$.

$x_{1}^{2}+3 x_{2}^{2}-75.16 \beta \geq-0.16$

$\left.2 x_{1}^{2}+13 x_{2}^{2}+3 x_{3}-175.19 \beta \geq-0.19\right\}$

Whose solution is assumed to be:

$\left(x_{1}^{S}, x_{2}^{S}, x_{3}^{S}\right)=(1.74,1.23,0.39),\left(f_{21}^{S}, f_{22}^{S}\right)=(7.56,17.81), \beta=0.1$

The TLDM solves his/her problem exactly like SLDMas follow:

\section{[3 $3^{\text {rd }}$ level]}

$\max _{3}\left(x_{1}, x_{2}, x_{3}\right)_{x_{3}}=\max _{x_{3}}\left[x_{1}+x_{2}+15 x_{3}^{2}, \quad 2 x_{1}^{2}+x_{2}^{2}+15 x_{3}^{2}\right]$

Subject to:

$\left(x_{1}, x_{2}, x_{3}\right) \in \mathrm{G}=\quad\left\{\left(x_{1} x_{2}, x_{3}\right)\right.$

$\left.x_{1}, x_{2}, x_{3} \geq 0\right\}$

$$
\begin{gathered}
x_{1}+x_{2}+x_{3} \leq 5 \\
x_{1}+2 x_{2}+x_{3} \leq 10 \\
2 x_{1}+x+x_{3} \leq 14
\end{gathered}
$$

$\left(f_{31}^{*}, f_{32}^{*}\right)=(375,375),\left(f_{31}^{-}, f_{32}^{-}\right)=(0,0)$

$\operatorname{Max} \gamma$,

Subject to :\{

$\left(x_{1}, x_{2}, x_{3}\right) \in G$, 
$x_{1}+x_{2}+15 x_{3}^{2}-375 y>=0$

$2 * x_{1}^{2}+x_{2}^{2}+15 x_{3}^{2}-375 \gamma>=0$

$\gamma \in[0,0.1]$. $\}$

Whose solution is assumed to be :

$\left(x_{1}^{T}, x_{2}^{T}, x_{3}^{T}\right)=(0.70,1.23,1.57),\left(f_{11}^{T}, f_{12}^{T}\right)=(38.90,39.46), \gamma=0.1$

Finally

1- Assume the FLDM's control decision is around 0 with tolerance 1.

2- Assume the SLDM's control decision is around 0 with the tolerance 1.

3- By using (12)-(17)theTchebycheff problem can be solved as follow:

$\operatorname{Max} \delta$,

Subject to:\{

$\left(x_{1}, x_{2}, x_{3}\right) \in G$,

$x_{1}-\delta>=1.6$

$-x_{1}-\delta>=-3.6$

$x_{2}-\delta>=0.23$

$-x_{2}-\delta>=-2.23$

$15 x_{1}^{2}+x_{2}^{2}+x_{3}+55.76 \delta>=47.31$

$6 x_{1}^{2}+3 x_{2}^{2}+19.2 \delta>=22.70$

$x_{1}^{2}+3 x_{2}^{2}+.54 \delta>=8.1$

$2 x_{1}^{2}+7 x_{2}^{2}+3 x_{3}+2.54 \delta>=20.35$

$x_{1}+x_{2}+15 x_{3}^{2}-33.64 \delta>=5.25$

$2 x_{1}^{2}+x_{2}^{2}+15 x_{3}^{2}-29.62 \delta>=9.84$

$\delta \in[.5,1]\}$

Whose is Compromise solution $X^{0}=(2.46,1.09,1.43), \delta=0.87$.

$\left(\mathrm{f}_{11}^{0}, \mathrm{f}_{12}^{0}\right)=(93.39,39.87)$

$\left(\mathrm{f}_{21}^{0}, \mathrm{f}_{22}^{0}\right)=(9.61,24.70)$

$\left(\mathrm{f}_{31}^{0}, \mathrm{f}_{32}^{0}\right)=(34.22,43.96)$

\section{CONCLUSION}

This paper proposed an algorithm to solve Multi-level multi-objective Quadratic programming problem, with fuzzy parameters in the objective functions, This algorithm used the concepts of tolerance membership function and multi-objective optimization at each level to develop a fuzzy Max-Min decision model for generating satisfactory solution after applying linear Ranking Method on trapezoidal fuzzy numbers in the objective function , This algorithm can be applied to problems when the fuzzy numbers in the constraint or in both the objective function and the constraint

\section{REFERENCE}

1. R. E. Bellman andL.A.Zadeh, "Decision- making in a fuzzy environment, Management Sciences", 17(4) (1970)141-164.

2. O.E.Emam, "A fuzzy approach for bi-level integer nonlinear programming problem, AppliedMathematics and Computations", 172 (2006) 62-71.

3. M.S.Osman, M.A. Abo-Sinna, A. H.Amer and O. E.Emam," A multi-level non-linear multi-objective under fuzziness, AppliedMathematics and Computations", 153 (2004) 239-252.

4. E.A. Youness, O.E.Emam and M.S. Hafez," Simplex method for solving bi-level linear fractional integer programming problems with fuzzy numbers", International Journal of Mathematical Sciences and Engineering Applications, 7 (2013) 351363.

5. . DP. Dash and R. B. Dash, "Solving Fuzzy Multi- Objective Non-linear Programming Problem Using Fuzzy Programming Technique”, International Journal of Engineering Science and Innovative Technology, 2 (2013)137-143. 
6. S. H. Nasseri," Quadratic Programming with Fuzzy Numbers: A Linear Ranking Method”, Australian Journal of Basic and Applied Sciences, 4(8) (2010) 3513-3518.

7. Z. A. Kanaya,“An Interactive Method for Fuzzy Multi-objective Nonlinear Programming Problems” ,JKAU,22(1) (2010 ) 103-112.

8. S.T. Liu, "Quadratic programming with fuzzy parameters: A membership function approach, Chaos", Solitons and Fractals, 40 (2009) 237-245.

9. O. M. Saad,M. Sh. Biltagy and T. B. Farag, "An Algorithm for Multi- Objective IntegerNonlinear Fractional Programming Problemunder Fuzziness”, Gen. Math. Notes, 2(2011) 1-17.

10. F .A. Adnanand I. H Alkanani, "Ranking Function Methods For Solving Fuzzy Linear Programming Problems", Mathematical Theory and Modeling,4(4) (2014)65-72.

11. H. Katagiri, K. Kato, T.Uno,"Bi-level Linear Programming Problems with Quadratic Membership Functions of FuzzyParameters", Proceedings of the International MultiConference of Engineers and Computer Scientists, 2(2013) 10311035 .

Osama E. Emam is Associate professor of information systems at Faculty of Computer Science and Information, Helwan University. He received the Ph.D. degree in operations research at Helwan University. His research interest in the multi- level

Amany M. Abdo is lecturer of the Department of Information Systems at Helwan University, where she has been since 1996. She received a B.S.c from Helwan University in 2000, and an M.S.c from the Faculty of Computers and Information at Helwan in 2004. She received her Ph.D. in Information Systems from Helwan University in 2010. Her research interests center on improving the understanding, design, and performance of information systems, mainly through the application of data mining, databases, and performance evaluation

Noha M. Bekhit is a Demonstrate at New Cairo Academy, Higher Institute for computer science and information systems also was Demonstrate at El Salam higher institute for Engineering and technology (S.H.I). She is a Postgraduate Student and Research Assistant in in the Faculty of Computers and Information, Helwan University where she received her bachelor degree in Information Systems. Her research interests are in fuzzy multi-level.
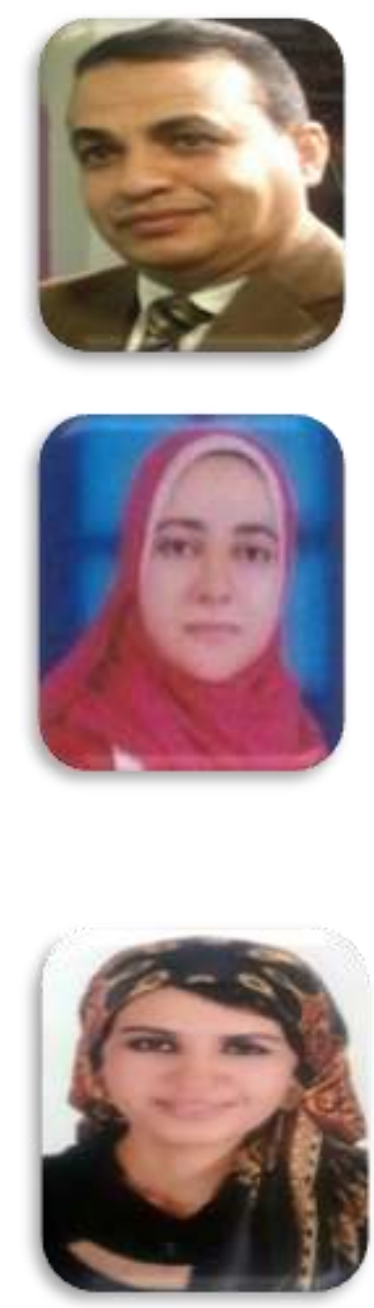